\title{
Synchronized epiaortic two-dimensional and color Doppler echocardiographic guidance enables routine ascending aortic cannulation in type A acute aortic dissection
}

\author{
Yoshito Inoue, MD, ${ }^{\mathrm{a}}$ Ryuichi Takahashi, MD, ${ }^{\mathrm{b}}$ Toshihiko Ueda, $\mathrm{MD},{ }^{\mathrm{c}}$ and Ryohei Yozu, $\mathrm{MD}^{\mathrm{d}}$
}

\begin{abstract}
Objectives: Preference for arterial inflow during surgery for type A acute aortic dissection remains controversial. Antegrade central perfusion prevents malperfusion and retrograde embolism, and the ascending aorta provides arterial access for rapid establishment of systemic perfusion, especially if there is hemodynamic instability. It has not been used routinely, however, because of the disruption caused to the aorta. We evaluated the safety and efficacy of routine cannulation of the dissected aorta for the repair of type A dissection.
\end{abstract}

\begin{abstract}
Methods: Surgical results were analyzed for 83 consecutive patients with type A acute aortic dissection between 2002 and 2009 . They were treated surgically by prosthetic graft replacement under hypothermic circulatory arrest. The ascending aorta was routinely cannulated using the Seldinger technique with epiaortic echocardiographic guidance; antegrade systemic perfusion was evaluated by color Doppler ultrasound.
\end{abstract}

Results: Systemic antegrade perfusion via the dissected ascending aorta was performed safely in all cases. There was no malperfusion or thromboembolism as a result of ascending aortic cannulation. Epiaortic 2-dimensional and color Doppler imaging provided real-time monitoring adequate for the placement and for proper systemic perfusion. There were 5 in-hospital deaths $(5 / 83=6.0 \%)$ and 8 strokes (preoperative $6 / 83=7.2 \%$, postoperative $2 / 83=2.4 \%)$. A total of 78 patients $(78 / 83=94 \%)$ were discharged and have been followed up without major adverse cardiac events for a mean duration of 31.8 months.

Conclusions: Ascending aortic cannulation is a simple and safe technique that provides a rapid and reliable route of antegrade central systemic perfusion in type A aortic dissection. (J Thorac Cardiovasc Surg 2011;141:354-60)

Preference for arterial inflow during surgery for type A acute aortic dissection remains controversial inasmuch as surgical mortality and morbidity is influenced by perfusion technique and the cannulation site for cardiopulmonary bypass. ${ }^{1}$ Ascending aortic cannulation in type A acute aortic dissection has been used mainly as a backup arterial inflow after the failure of primary peripheral cannulation attempts. ${ }^{2}$ This method of cannulation for aortic dissection has recently been revived, and occasional successful reports indicate that a dissected ascending aorta in fact remains an option for primary arterial inflow in type A aortic dissection. $^{3-5}$ This is because antegrade central perfusion may have a potential to prevent malperfusion and retrograde cerebral embolism. ${ }^{6}$ It is also effective for rapid establish-

From the Department of Cardiovascular Surgery, ${ }^{\text {a }}$ Hiratsuka, City Hospital, Kanagawa; the Department of Cardiovascular Surgery, ${ }^{\mathrm{b}}$ Saiseikai Utsunomiya Hospital, Tochigi; the Department of Cardiovascular Surgery, ${ }^{\mathrm{c}}$ Tokai University, Kanagawa; and the Department of Surgery, ${ }^{\mathrm{d}}$ Keio University School of Medicine, Tokyo, Japan

Disclosures: Authors have nothing to disclose with regard to commercial support.

Read at the 36th Annual Meeting of The Western Thoracic Surgical Association, Ojai, California, June 23-26, 2010.

Received for publication June 16, 2010; revisions received Oct 31, 2010; accepted for publication Nov 4, 2010.

Address for reprints: Yoshito Inoue, MD, Department of Cardiovascular Surgery, Hiratsuka City Hospital, 1-19-1 Minamihara, Hiratsuka, Kanagawa 254-0065, Japan (E-mail: yoshito_inoue@sky.plala.or.jp).

0022-5223/\$36.00

Copyright (c) 2011 by The American Association for Thoracic Surgery doi:10.1016/j.jtcvs.2010.11.010 ment of systemic perfusion, especially if there is hemodynamic instability. ${ }^{5}$

Regarding ascending aortic cannulation, there are several concerns: disruption of the cannulation site, risk of thromboembolism and extension of dissection, and doubt over the establishment of adequate antegrade systemic perfusion. It is also not yet clear whether antegrade perfusion through the ascending aorta would contribute to distal organ perfusion. ${ }^{7}$ If these issues were resolved, ascending aortic cannulation would be accepted as a reliable route of arterial access in type A aortic dissection. We therefore determined the efficacy and safety of a routine cannulation of the dissected aorta for the repair of type A aortic dissection, performed using the Seldinger technique and epiaortic 2-dimensional and color Doppler ultrasound.

\section{PATIENTS AND METHODS}

Surgical results were analyzed for 83 consecutive patients with Stanford type A acute aortic dissection between February 2002 and August 2009. The ascending aorta was routinely cannulated by the Seldinger technique with epiaortic echocardiographic guidance. Preoperative patient profiles are summarized in Table 1 . They were treated surgically by prosthetic graft replacement of the ascending aorta/hemiarch or total arch. Surgical treatment was performed within a mean of 2.3 days of dissection; 57 patients $(57 / 83=68.7 \%)$ were operated on within 24 hours of dissection. The institutional ethics committees approved this study and waived the need for patient consent. Preoperatively, there were 14 preoperative symptoms related to malperfusion of arch vessels, 4 instances of myocardial ischemia, and 5 of limb ischemia. 
Surgery was performed through a median sternotomy. The arterial inflow to the cardiopumonary bypass circuit was bifurcated in advance, one branch for the ascending aorta and the other for the femoral artery. Median sternotomy, to access the ascending aorta and prepare the femoral artery, was performed simultaneously by 2 surgeons to save time. Cardiopulmonary bypass was performed at a flow rate of $2.1 \mathrm{~L} \cdot \mathrm{min}^{-1} \cdot \mathrm{m}^{-2}$. The left ventricle was vented through the right superior pulmonary vein. Carbon dioxide was continuously infused into the surgical field at the flow rate of $2 \mathrm{~L} / \mathrm{min}$. Hypothermic circulatory arrest was established below $16^{\circ} \mathrm{C}$ at the blood temperature of the venous drainage cannula. Distal repair was performed first with open distal anastomosis, using a woven Dacron onebranch graft (Boston Scientific Corp. Natick, Mass) with felt strip reinforcement. In cases that required total arch reconstruction, arch branches were reconstructed first so as to minimize the duration of circulatory arrest. On completion of distal repair, retrograde brain perfusion and retrograde femoral perfusion were performed to flush out air and atheromatous debris within arch vessels and the descending aorta. Antegrade systemic perfusion and rewarming were restarted through the side branch of the graft. Blood cardioplegic solution was selectively administered into the coronary ostia, and proximal repair was subsequently performed. The native aorta was not clamped during the operation, although the prosthetic graft was, to prevent clamp-induced malperfusion. Aortic valve resuspension was performed in 36 cases, composite graft replacement in 2 cases, and concomitant coronary artery bypass grafting in 3 cases. Gelatin-resorcin-formalin biological glue (Caridal SA, Saint-Etienne, France) was used for proximal repair in 40 cases.

Ascending aortic cannulation for acute type A aortic dissection was performed according to the following protocol. To decompress the cannulation site and secure safe cannulation through dissected fragile adventitia, we lowered mean arterial pressure before cannulation by the initiation of a femoral artery and right atrial circuit. Dual-dynamic display mode epiaortic ultrasonography was then used, with 2-dimensional cross-sectional and color flow imaging (Prosound SSD-3500 and Linear Array Probe; UST5534T-7.5: Aloka Co Ltd, Tokyo, Japan, or iE33: Philips Medical Systems, Bothell, Wash) to guide a puncture through a single pledget-supported 4-0 polypropylene mattress suture placed where subsequent reconstruction is not required. The true and false channels were detected by epiaortic echocardiography in combination with the anatomic information from preoperative computed tomography. The true lumen was identified by systolic expansion and diastolic collapse, as well as by the presence of antegrade systolic flow in cases of perfused false lumen. In cases of thrombosed false lumen with absence of flow, the true lumen was identified simply by the presence of the color flow signal. After the arterial entry catheter was placed within the true channel, a guidewire was advanced into the true lumen, and the aortic cannulation site was dilated in a stepwise fashion by 3 -staged spindle-shape tapered dilators. The heparin-coated flexible thin-walled cannula (Flexmate cannulas: Toyobo, Ltd, Osaka, Japan, or Fem-Flex II arterial cannulas: Edwards Life Sciences Research Medical, Midvale, Utah) was then inserted over the guidewire. The apical location and antegrade systemic perfusion from the ascending aortic cannula were confirmed by epiaortic echocardiography with synchronized B-mode and color flow imaging (Figure 1).

Cannulation sites and the techniques of puncture were modified as necessary to take account of preoperative computed tomography and epiaortic echocardiographic observation of the false lumen and the type and range of disruption. A mattress suture was placed at the left lateral side of the ascending aorta, sometimes near the posterior lesser curve of the proximal arch, where the true lumen could be reached directly or within the shortest distance (Figure 2, $A$ and $B$ ). In cases of thrombosed false lumen, the aorta was punctured at nearly $90^{\circ}$ to the short axis, and the cannula was inserted deeply enough to be located with certainty within the true lumen (Figure 2, $C$ ). Conversely, in cases of perfused false lumen with dynamic true channel collapse and presence of dissection at the left lateral side of ascending aorta, puncture was made at a shallow angle to the direction of longitudinal
TABLE 1. Preoperative patient profiles

\begin{tabular}{lc}
\hline No. of patients & 83 \\
Age (y), median $\pm \mathrm{SD}$ & $62.2 \pm 10.2$ \\
Male/female & $44 / 39$ \\
Ascending aorta diameter (mm) & $51.5 \pm 6.9$ \\
Aortic regurgitation (no.) & 30 \\
Cardiac tamponade (no.) & 22 \\
Rupture (no.) & 5 \\
Shock (no.) & 11 \\
Malperfusion (no.) & 21 \\
\hline
\end{tabular}

$S D$, Standard deviation.

axis toward aortic arch to be sure of reaching the center of the narrowed true lumen (Figure 2, D).

\section{RESULTS}

The cannulation to the ascending aorta and systemic antegrade perfusion via the dissected aorta were successfully performed in all cases; in no case was revision of the aortic cannulation necessary. Neither malperfusion nor thromboembolism occurred as a result of ascending aortic cannulation. With decompression of the aortic pressure in advance (mean arterial pressure $51.4 \pm 12.3 \mathrm{~mm} \mathrm{Hg}$ ), the adventitia of the dissected aorta readily supported the aortic cannulaion. Any local hemorrhage in the Seldinger technique was easily controlled by a fingertip. Tourniquet compression with a pledget-supported mattress suture totally restrained bleeding after cannulation.

There were 5 in-hospital deaths $(5 / 83=6.0 \%)$. The causes of death were low output syndrome owing to preoperative acute myocardial infarction $(n=2)$, graft infection $(\mathrm{n}=1)$, sepsis $(\mathrm{n}=1)$, and duodenal bleeding $(\mathrm{n}=1)$. There were 8 strokes, including 6 preoperative strokes $(6 / 83=7.2 \%)$ and 2 postoperative strokes $(2 /$ $83=2.4 \%$ ). Among 6 patients with preoperative strokes, 4 patients had a complicated permanent neurologic deficit and 2 patients showed recovery from the symptom by the time of discharge. Of these 2 patients with postoperative neurologic dysfunction, 1 patient had a temporary neurologic disorder and the other had hypoxic-ischemic encephalopathy, having once recovered without neurologic dysfunction, owing to acute pulmonary embolism on the eighth day after the operation. A total of 31 patients (31/ $83=37.3 \%$ ) required ventilatory support for more than 4 days, including 5 patients with pneumonia. Eight patients had acute renal failure, of whom 7 required temporary hemodialysis. A total of 78 patients $(78 / 83=94.0 \%)$ were discharged and have been monitored with periodic chest computed tomography without major adverse cardiac events for a mean of 31.8 months. Postoperative examination by computed tomography did not find any subsequent extension of the false lumen owing to ascending aortic cannulation. 


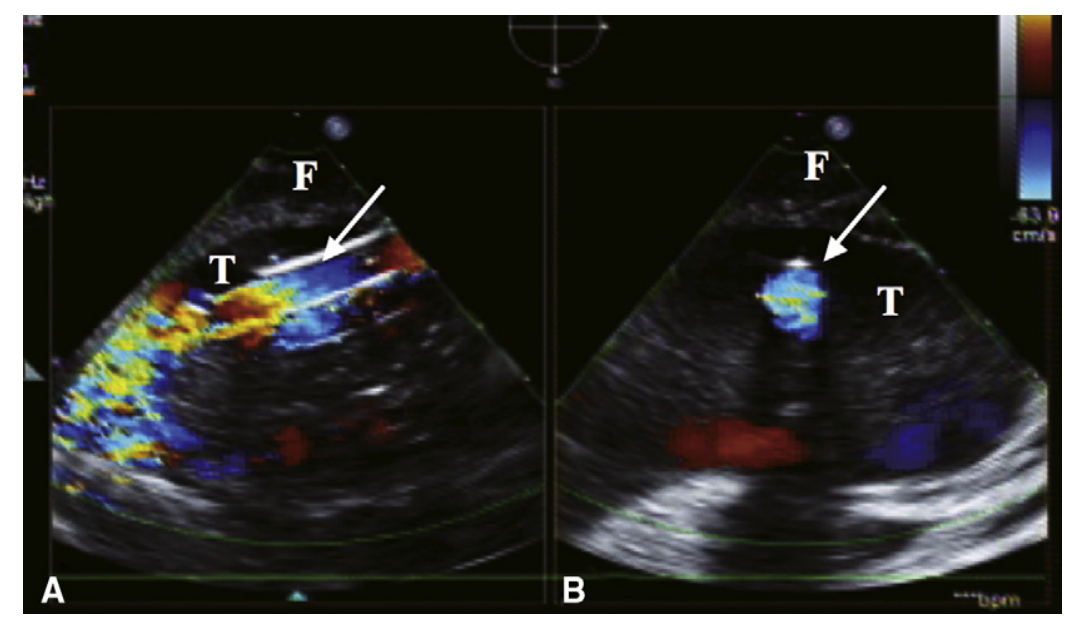

FIGURE 1. Dual display of color flow imaging; long axis (A) and short axis (B) of inflow cannula located in true lumen of the ascending aorta (arrow) and color Doppler image of antegrade systemic perfusion. $T$, True lumen; $F$, false lumen.

Open distal anastomosis was started at an average of 46 minutes after skin incision; the time from skin incision to initiation of cardiopulmonary bypass was $23.7 \pm 6.7$ minutes, and hypothermic circulatory arrest was established $22.5 \pm 5.4$ minutes after initiation of cardiopulmonary bypass. The duration of cardiopulmonary bypass was $134.8 \pm 50.1$ minutes, the duration of deep hypothermic circulatory arrest was $25.5 \pm 5.1$ minutes, and that of the operation was $280.2 \pm 91.9$ minutes. The mean esophageal temperature at hypothermic arrest was $14.3^{\circ} \mathrm{C} \pm 0.31^{\circ} \mathrm{C}$.

Throughout this procedure, epiaortic ultrasonographic color flow imagery confirmed antegrade perfusion to the aortic arch branches. Ascending aortic perfusion was established within $4.9 \pm 2.6$ minutes after the femoral cannulation. During this interval, only the antegrade pulsatile arterial blood flow from the beating heart was observed at the transverse arch by epiaortic color Doppler imaging. After ascending aortic cannulation, only antegrade flow was observed. In recent cases, femoral inflow was clamped after ascending aortic cannulation to establish single antegrade perfusion.

The left lateral to posterior lesser curve of the ascending aorta and arch was less frequently dissected, and even if dissected, the gap between the intima and adventitia was small (Figure 2, $A$ and $B$ ). The shape of the dissection of the ascending aorta was semicircular (Figure $2, B$ ) in 68 cases $(68 / 83=81.9 \%)$ and circular (Figure $2, A)$ in 15 cases $(15 / 83=18.1 \%)$. Dissection of the left lateral lesser curve of the ascending aorta adjacent to the pulmonary artery was observed in only $19(22.9 \%)$ of the 83 cases studied. There were 38 cases of thrombosed false lumen $(38 / 83=45.8 \%)$ and 45 cases of perfused false lumen $(45 / 83=54.2 \%)$. The intimal tear was observed within the ascending aorta or arch in 69 cases $(69 / 83=83.1 \%)$.

Surgical inspection of the ascending aorta during circulatory arrest was undertaken in all cases. In the patients with thrombosed false lumen, there was no false lumen cannulation and no dislodgment of the thrombus was observed inside the false lumen. Furthermore, no cerebral or other thromboembolism was observed in this group. In patients with perfused false lumen, there was inadvertent false lumen cannulation in 6 of the first 42 patients. Patients with false lumen cannulation had a DeBakey type $I(n=5)$ or type II $(\mathrm{n}=1)$ dissection with a patent false lumen, in which the intimal tear was located within the ascending aorta adjacent to the inflow cannula. In these cases, the true luminal antegrade flow was observed via a large intimal fenestration. One case of false lumen cannulation was complicated with brain infarction, but this neurologic injury began before surgery, and its symptom did not change after surgery. There was no neurologic disorder in the other 5 patients. In these 6 patients, there were no cases of malperfusion or subsequent extension of the false lumen. After modification of the puncture technique with proficiency gained in routine dual-dynamic mode epiaortic echocardiographic guidance, no false lumen cannulation occurred in the last 41 patients.

Dual-display mode epiaortic 2-dimensional and color Doppler imaging provided synchronized real-time monitoring, adequate for the placement and proper perfusion of ascending aorta cannulation, as well as the location of the intimal tear and perfusion to the arch vessels. Malperfusion evoked by valvelike motion of the intimal flap was observed (Figure 3, $A$ and $B$ ). Ascending aortic cannulation was also guided by real-time epiaortic 3-dimensional imaging, which provided dynamic spatial anatomic landmarks of the dissected aorta (Figure 4).

It was also possible to monitor the transition in flow dynamics and intimal flap motion. In cases of thrombosed false lumen, the dimension of the true channel remains constant regardless of the cardiac cycle during systemic cooling, inasmuch as the adventitia and intima are bonded by a tenacious thrombus layer. In cases of perfused false 
lumen, however, the movement of the intimal flap changed completely during cooling. During the period of pulsatile flow, intense motion of the intimal flap was observed with systolic expansion, and dynamic collapse of the true lumen was evoked by retrograde flow of the false lumen (Figure 5, $A$ and $B$ ). After pulsatile flow ceased, the dynamic motion converted gradually to a cessation of diastolic collapse and static intimal flap motion owing to pressure equilibrium across the entry tear. The diameter of the true and false lumina remained constant under nonpulsatile antegrade perfusion during cooling.

\section{DISCUSSION}

Ascending aortic cannulation with the Seldinger technique, in combination with epiaortic 2-dimensional and color Doppler echocardiographic guidance, facilitated systemic antegrade perfusion in a safe and reproducible manner. Our results show that ascending aortic cannulation is more than merely a backup arterial inflow in the cases of failure to establish a primary peripheral cannulation in type A acute aortic dissection.

The ascending aorta offers several advantages as an alternative access for cannulation. It is suitable for rapid establishment of cardiopulmonary bypass in cases of hemodynamic instability, because it is easy to access, ${ }^{8}$ and it is also useful in cases of dissected axillary artery or diseased iliac or femoral artery. There is no limitation on the diameter of inflow cannula, so that a large-diameter cannula can be used if the patient's body weight requires it. Also, ascending aortic cannulation does not require subsequent repair of the cannulation site.

In particular, ascending aortic cannulation provides natural antegrade systemic perfusion ${ }^{9}$ and may be able to minimize the incidence of malperfusion syndromes during cardiopulmonary bypass for the repair of aortic dissections. Epiaortic 2-dimensional and color Doppler mapping revealed conversion of the intimal deflection and the transition in flow distribution during systemic cooling. Antegrade systemic perfusion effectively arrested intimal flap motion by prompt cooling and subsequently prevented collapse of the true lumen. Inasmuch as malperfusion is a result of distention of the false lumen arising from pressure difference between the 2 lumina, ${ }^{2}$ decompression of the false lumen and pressure equilibrium across the intimal fenestration may permit adequate antegrade systemic perfusion through the true (or mixed true and false) lumen. ${ }^{10}$ This observation suggests that rapid cooling and quick conversion of intimal flap motion by central cannulation may indeed be contributing to bailout from underlying organ malperfusion. ${ }^{11}$ In restoring neutral intimal flap deviation and pressure equilibrium between 2 channels, aortic clamps should be avoided. ${ }^{12}$

There is a possible concern over malperfusion in cases of false lumen cannulation. ${ }^{13}$ False lumen perfusion occurs regardless of the location of arterial inflow, ${ }^{14}$ making it impor-
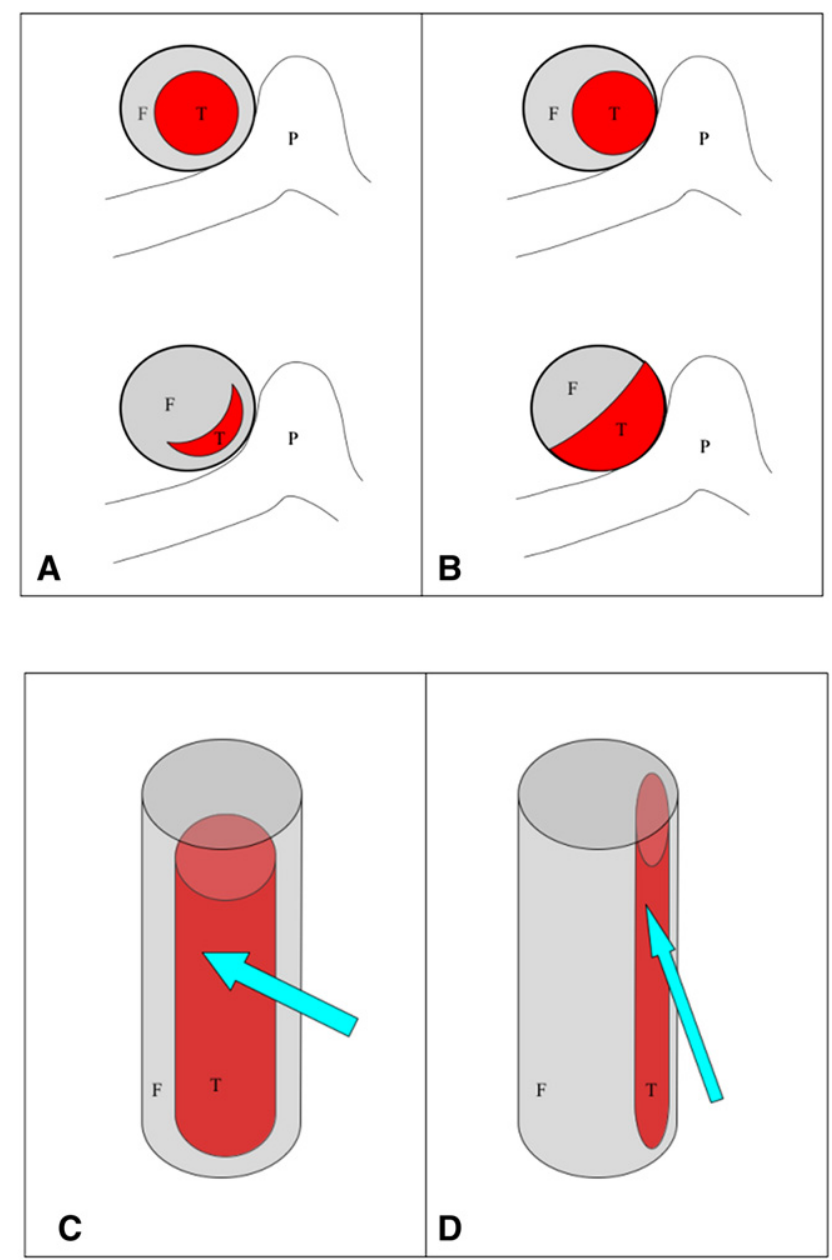

FIGURE 2. Computed tomographic scheme of dissection. A, Circular dissection: circumferential abnormal gap between intima and adventitia. B, Semicircular dissection: presence of intact portion without dissection, mostly at left lateral lesser curve, $\mathrm{C}$, Aorta was punctured nearly toward short axis in cases of thrombosed false lumen deeply to reach inside the true lumen. D, Puncture was performed at a shallow angle in the direction of longitudinal axis toward aortic arch to reach the narrowed true lumen in cases of perfused false lumen. $T$, True lumen; $F$, false lumen. $P$, pulmonary artery.

tant to monitor the actual flow distribution inside the aorta. The color Doppler ultrasonographic observation during cardiopulmonary bypass demonstrated adequate true lumen or mixed true and false lumen perfusion. This might indicate that, in cases of perfused false lumen, antegrade nonpulsatile flow through false lumen inflow under hypothermia might not always cause malperfusion so long as a large intimal fenestration is located adjacent to the inflow cannula in the ascending aorta. ${ }^{15,16}$ The large intimal fenestrations may communicate flow between the false and true lumina and may therefore have prevented malperfusion. This kind of observation has been documented in several articles with acceptable results in cases in which mixed antegrade true and false lumen perfusion from the ascending aorta has 


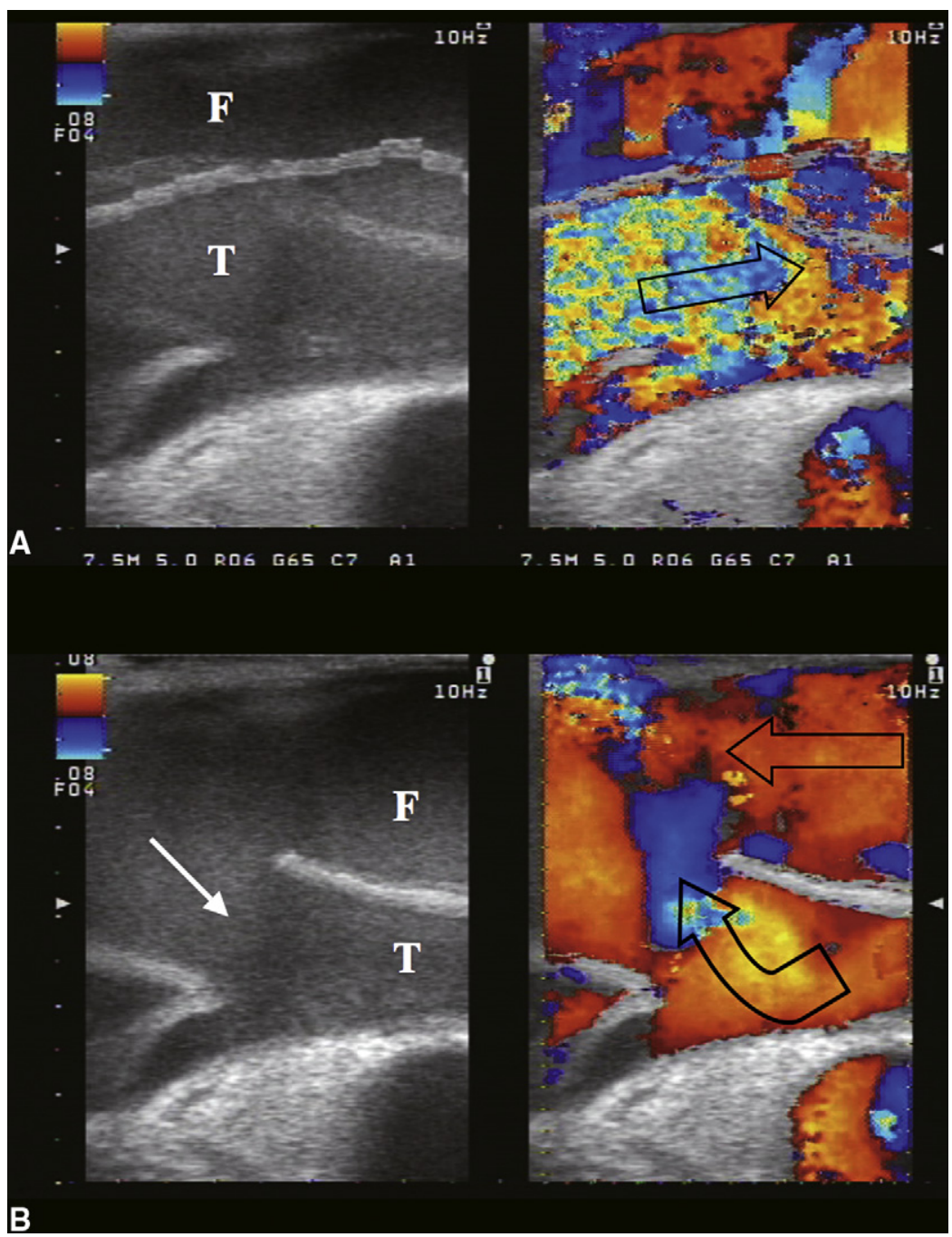

FIGURE 3. Dual display of epiaortic 2-dimensional (left panel) and color flow imaging (right panel) provided dynamic collapse and systolic expansion of the true lumen, evoked by valvelike motion of intimal flap of ascending aorta in a case of malperfusion (right, cranial side.) A, Primary entry tear closes during systole and antegrade true lumen flow (black arrow) is recognized. B, During diastole, primary tear opens (white arrow) and retrograde flow (black arrow) from both lumina runs toward the false lumen of the aortic root. $T$, True lumen; $F$, false lumen.

been used for backup after primary cannulation failure, ${ }^{2,16,17}$ resulting in the restoration of adequate systemic perfusion. However, the safety of central cannulation should be represented by the establishment of true channel antegrade perfusion. Modification of puncture technique with proficiency gained in routine dual-dynamic mode epiaortic echocardiographic guidance could eliminate false channel cannulation.

Cannulation to the true lumen was simple in cases of thrombosed false lumen, inasmuch as the adventitia and intima are bonded by a tenacious thrombus layer for which the color flow signal is absent; also, the dimension of the true channel remains constant regardless of the cardiac cycle. True lumen cannulation involved a learning curve, however, in cases of perfused false lumen with intense flap motion or collapsed narrow true lumen. To cannulate the true lumen reliably in these cases of circumferential dissection, one must be proficient in Seldinger cannulation using epiaortic 2-dimensional and color Doppler mapping; fortunately, this is simple. It is also helpful not to perform cannulation until bradycardia during cooling, or to induce bradycardia pharmacologically, to facilitate recognition of the true channel.

Besides femoral cannulation, there may be other means of obtaining decompression of the aorta, such as pharmacologic means as well as hypovolemic decompression by venous drainage from the right atrium. They may be easier and could be one of the options for some cases. However, it might be risky to induce intentional hypotension before cardiopulmonary bypass in some cases, such as the cases in which there is underlying preoperative neurologic deficiency or coronary malperfusion, which could be acutely worsened by hypoperfusion. It would be safer to establish 


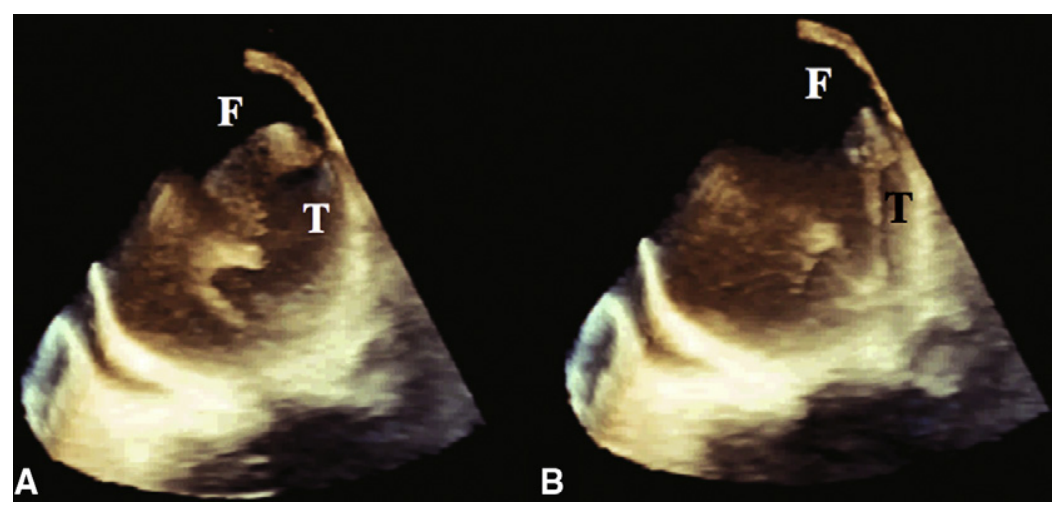

FIGURE 4. Three-dimensional image of systolic expansion (A) and dyastolic collapse (B) of the true lumen of the ascending aorta in perfused false lumen during the period of pulsatile flow. $T$, Tue lumen; $F$, false lumen.

cardiopulmonary support in advance to prepare for any situation that might have contributed to the low mortality and morbidity to some extent. Also, femoral perfusion was effective to flush out air and atheromatous debris within the descending aorta on completion of distal repair to avoid distal organ embolism.

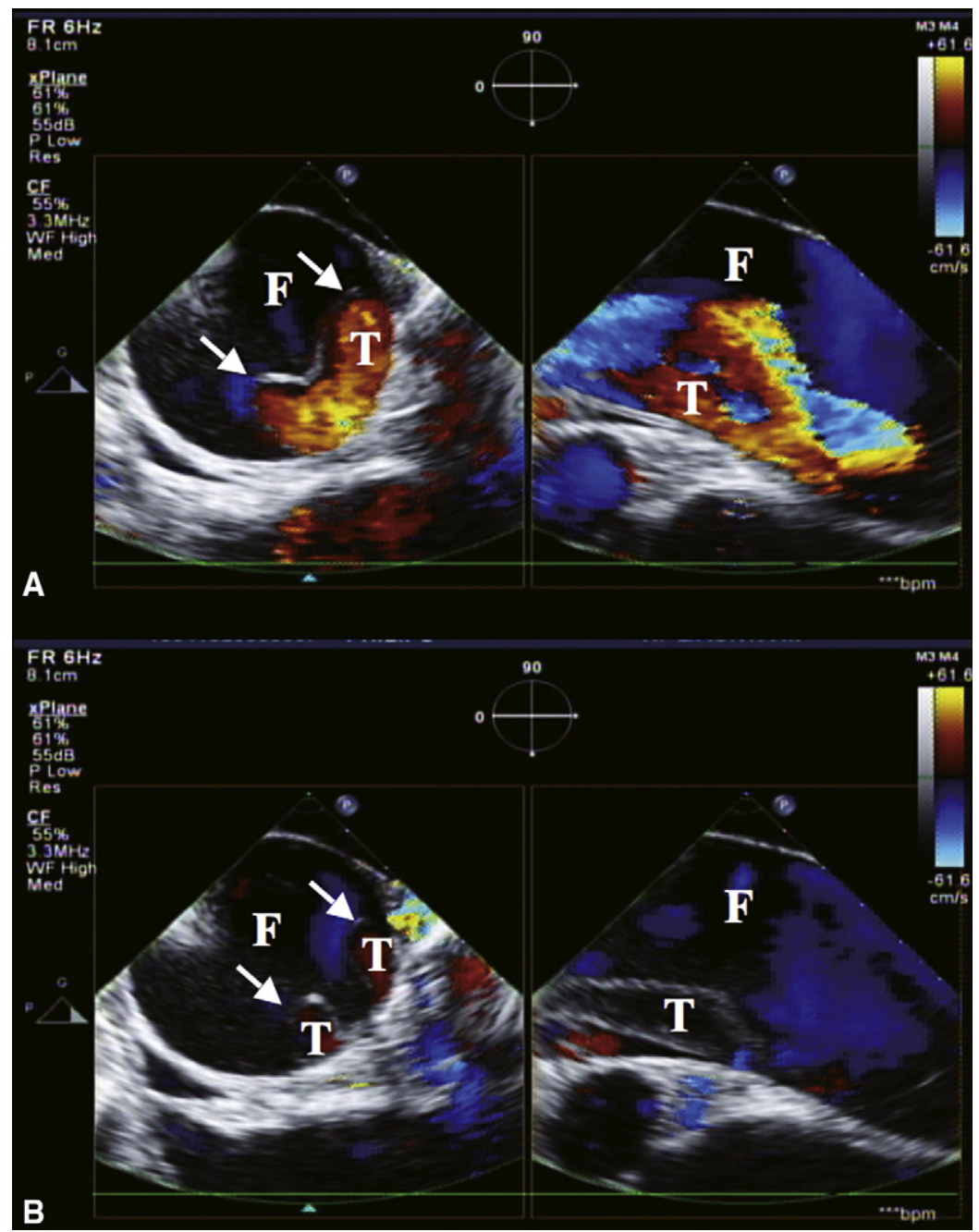

FIGURE 5. Dual display of short-axis (left panel) and long-axis (right panel) color flow imaging. A, During systole, color Doppler signal shows antegrade systemic perfusion within the true lumen (arrow indicates intimal flap). B, During diastolic phase, true lumen collapses and color Doppler signal is extremely decreased in the true lumen. $T$, True lumen; $F$, false lumen. 
A limitation of this study is that comparison was not made with femoral and axillary artery cannulation. Surgical results in this study may be influenced not only by this procedure but also by a patient's preoperative status and risk factors for cardiovascular diseases. Transesophageal echocardiography was not performed to guide cannulation because epiaortic echocardiography provided adequate information on the ascending aorta and arch. Peripheral cerebral blood flow during cardiopulmonary bypass was not evaluated. The number of patients may be insufficient for us to conclude that ascending aortic cannulation can be performed in every patient with type A aortic dissection. Other modes of cannulation may be safe as well. There may be a situation in which malperfusion cannot be prevented regardless of cannulation site.

In conclusion, ascending aortic cannulation is a simple and safe technique that provides a rapid and reliable route for antegrade central systemic perfusion in type A acute aortic dissection. Prompt establishment of antegrade central perfusion and rapid systemic cooling by ascending aortic cannulation may contribute to effective bailout from malperfusion.

\section{References}

1. Strauch JT, Spielvogel D, Lauten A. Axillary artery cannulation: routine use in ascending aorta and aortic arch replacement. Ann Thorac Surg. 2004;78:103-8.

2. Borst HG, Heinemann M, Stone CD. Organ ischemia. In: Ross A, Dickinson BL, eds. Surgical treatment of aortic dissection. New York: Churchill Livingstone; 1996:265-6.

3. Lijoi A, Scarano F, Dottori V, Parodi E, Casall G, Bartolozzi F. Stanford type A aortic dissection. A new surgical approach. Tex Heart Inst J. 1998;25: 65-7.
4. Minatoya K, Karck M, Szpakowski E, Harringer W, Haverich A. Ascending aortic cannulation for Stanford type A acute aortic dissection: another option. J Thorac Cardiovasc Surg. 2003;125:952-3.

5. Conzelmann LO, Kayhan N, Mehlhorn U, Weigang E, Dahm M, Vahl CF. Reevaluation of direct true lumen cannulation in surgery for acute type A aortic dissection. Ann Thorac Surg. 2009;87:1182-6.

6. Svensson LG, Blacksone EH, Rajeswaran J, Sabik JF III, Lytle BW, Gonzalez-Stawinski G, et al. Does the arterial cannulation site for circulatory arrest influence stroke risk? Ann Thorac Surg. 2004;78:1274-84.

7. Robicsek F, Guarino RL. Compression of the true lumen by retrograde perfusion during repair of aortic dissection. J Cardiovasc Surg. 1985;26:36-40.

8. Inoue Y, Ueda T, Taguchi S, Kashima I, Koizumi K, Takahashi R, et al. Ascending aorta cannulation in acute type A aortic dissection. Eur J Cardiothorac Surg. 2007;31:976-81.

9. Reece TB, Tribble CG, Smith RL, Singh RR, Stiles BM, Peeler BB, et al. Central cannulation is safe in acute aortic dissection repair. J Thorac Cardiovasc Surg. 2007; 133:428-34.

10. Voci P, Testa G, Tritapepe L, Menichetti A, Caretta Q. Detection of false lumen perfusion at the beginning of cardiopulmonary bypass in patients undergoing repair of aortic dissection. Crit Care Med. 2000;28:1841-6.

11. Van Arsdell GS, David TE, Butany J. Autopsies in acute type A aortic dissection. Surgical implication. Circulation. 1998;98(19 Suppl):II299-304.

12. David TE, Armstrong S, Ivanov J, Barnard S. Surgery for acute type A aortic dissection. Ann Thorac Surg. 1999;67:1999-2001.

13. Khaladj N, Shrestha M, Peterss S, Strueber M, Karck M, Pichlmaier M, et al. Ascending aortic cannulation in acute aortic dissection type A: the Hanover experience. Eur J Cardiothorac Surg. 2008;34:792-7.

14. Yavuz S, Goncu MT, Turk T. Axillary artery cannulation for arterial inflow in patients with acute dissection of ascending aorta. Eur J Cardiothorac Surg. 2002;22:313-5.

15. Estrera AL, Garami Z, Miller CC, Sheinbaum R, Huynh TT, Porat EE, et al. Cerebral monitoring with transcranial Doppler ultrasonography improves neurologic outcome during repairs of acute type A aortic dissection. J Thorac Cardiovasc Surg. 2005;129:277-85.

16. Kamiya H, Kallenbach K, Halmer D, Ozsoz M, Ilg K, Lichtenberg A, et al. Comparison of ascending aorta versus femoral artery cannulation for acute aortic dissection type A. Circulation. 2009;120:S282-6.

17. Parr GV, Manley NJ, Williams DR, Montesano RM. Obstruction of the true lumen during retrograde perfusion of type I aortic dissections: a simplified solution. Ann Thorac Surg. 1980;30:495-8. 